\title{
BMJ
}

\section{Protective efficacy of co-trimoxazole prophylaxis against malaria in HIV exposed children in rural Uganda: a randomised clinical trial}

\author{
Taylor G Sandison, acting instructor, ${ }^{1}$ Jaco Homsy, assistant professor, ${ }^{2}$ Emmanuel Arinaitwe, study \\ coordinator, ${ }^{3}$ Humphrey Wanzira, medical officer, ${ }^{3}$ Abel Kakuru, medical officer, ${ }^{3}$ Victor Bigira, medical \\ officer, ${ }^{3}$ Julius Kalamya, PMTCT coordinator, ${ }^{4}$ Neil Vora, medical student, ${ }^{5}$ James Kublin, associate \\ professor, ${ }^{6,7}$ Moses R Kamya, associate professor, ${ }^{8}$ Grant Dorsey, associate professor, ${ }^{5}$ Jordan W Tappero, \\ acting deputy director ${ }^{9}$
}

${ }^{1}$ Department of Medicine, University of Washington, UW/ FHCRC Clinical Research,

Box 358080, Seattle, WA 981958080 USA

${ }^{2}$ Institute for Global Health, University of California, San Francisco, USA

${ }^{3}$ Infectious Diseases Research Collaboration, Kampala, Uganda

${ }^{4}$ Centers for Disease ControlUganda, Entebbe, Uganda

${ }^{5}$ Department of Medicine,

University of California, San Francisco, USA

${ }^{6}$ Vaccine and Infectious Disease Institute, Fred Hutchinson Cancer Research Center, Seattle, USA

${ }^{7}$ Department of Global Health, University of Washington, Seattle, USA

${ }^{8}$ Department of Medicine, Makerere University Medical School, Kampala, Uganda

${ }^{9}$ Global AIDS Program, Centers for Disease Control and Prevention, Atlanta, USA

Correspondence to: T Sandison tgsand@u.washington.edu

TGS and JH contributed equally to the preparation of the manuscript.

Cite this as:BMJ 2011;342:d1617 doi:10.1136/bmj.d1617

\section{ABSTRACT}

Objective To evaluate the protective efficacy of co-trimoxazole prophylaxis against malaria in HIV exposed children (uninfected children born to HIV infected mothers) in Africa.

Design Non-blinded randomised control trial Setting Tororo district, rural Uganda, an area of high malaria transmission intensity

Participants 203 breastfeeding HIV exposed infants enrolled between 6 weeks and 9 months of age Intervention Co-trimoxazole prophylaxis from enrolment until cessation of breast feeding and confirmation of negative HIV status. All children who remained HIV uninfected $(n=185)$ were then randomised to stop co-trimoxazole prophylaxis immediately or continue co-trimoxazole until 2 years old.

Main outcome measure Incidence of malaria, calculated as the number of antimalarial treatments per person year. Results The incidence of malaria and prevalence of genotypic mutations associated with antifolate resistance were high throughout the study. Among the 98 infants randomised to continue co-trimoxazole, 299 malaria cases occurred in 92.28 person years (incidence 3.24 cases/person year). Among the 87 infants randomised to stop co-trimoxazole, 400 malaria cases occurred in 71.81 person years (5.57 cases/person year). Co-trimoxazole prophylaxis yielded a $39 \%$ reduction in malaria incidence, after adjustment for age at randomisation (incidence rate ratio $0.61(95 \% \mathrm{Cl} 0.46$ to $0.81), P=0.001)$. There were no significant differences in the incidence of complicated malaria, diarrhoea, pneumonia, hospitalisations, or deaths between the two treatment arms.

Conclusions Co-trimoxazole prophylaxis was moderately protective against malaria in HIV exposed infants when continued beyond the period of HIV exposure despite the high prevalence of Plasmodium genotypes associated with antifolate resistance.

Trial registration Clinical Trials NCT00527800

\section{INTRODUCTION}

HIV and malaria are two of the deadliest infectious diseases, together accounting for more than four million deaths annually worldwide and more than a million deaths in children aged less than 5 years in subSaharan Africa. ${ }^{12}$ Malaria prevention is especially important in children infected with HIV or exposed to HIV (uninfected children born to HIV infected mothers) given the increased susceptibility of these children to severe illness and death. ${ }^{3-5}$ The primary method of malaria prevention in young children is the use of bed nets treated with insecticide. However, there is now renewed interest in chemoprevention, the use of antimalarial drugs to prevent disease. One option for chemoprevention is the use of daily cotrimoxazole prophylaxis, based on evidence from observational cohorts of HIV infected children and a single randomised control trial of HIV uninfected children showing that co-trimoxazole prophylaxis protects children from malaria. ${ }^{6-9}$

The World Health Organization (WHO) recommends placing all infants of HIV infected mothers on co-trimoxazole prophylaxis starting at 6 weeks of age until cessation of breast feeding and exclusion of HIV infection. ${ }^{10}$ This guideline stems from evidence that cotrimoxazole prophylaxis protects HIV infected children from common opportunistic infections, ${ }^{11-13}$ including malaria. ${ }^{679}$ However, there are no studies on the protective efficacy of co-trimoxazole prophylaxis against malaria among HIV exposed, uninfected infants. More than two million infants are born to HIV infected African mothers each year, with more than $90 \%$ remaining uninfected throughout breast feeding. ${ }^{14-16}$ To exclude HIV infection in these children, HIV DNA testing by polymerase chain reaction (PCR) is required because of the persistence of maternal HIV antibodies in infant blood until 18 months of age. However, access to PCR testing remains extremely limited in most resource poor settings, and so large numbers of HIV exposed children throughout 
Africa continue taking co-trimoxazole prophylaxis until 18 months old, when exclusion of HIV infection by antibody testing becomes possible.

We conducted a randomised clinical trial to examine whether prolonged daily co-trimoxazole prophylaxis beyond the period of breast feeding provides sustained protective efficacy against malaria in HIV uninfected children born to HIV infected mothers in an area of high malaria transmission intensity in rural Uganda.

\section{METHODS}

\section{Study setting and enrolment of participants}

The study was conducted in Tororo, a rural setting located in southeastern Uganda, where malaria is holoendemic and the inoculation rate from insect bites is 591 infective bites per person year. ${ }^{17}$ We used convenience sampling to enrol a cohort of HIV exposed infants from the Mother and Child Health Clinic of Tororo District Hospital and the AIDS Support Organization (TASO) between August 2007 and April 2008. Mothers were not enrolled in the study, and their HIV infection was confirmed by serology through a national HIV testing programme.

Infants' eligibility criteria included age 6 weeks to 9 months, documented HIV uninfected status of infant and HIV infected status of mother, current breast feeding, residence within $30 \mathrm{~km}$ radius of the study clinic, parent's or guardian's agreement to come to the study clinic for any febrile episode or other illness and to avoid medications administered outside the study protocol, absence of active medical problem requiring inpatient evaluation at the time of screening, and parent's or guardian's informed consent. All infant participants underwent a standardised clinical history and a physical examination at enrolment including thick and thin blood smears and a complete blood count. In addition, all participants were provided with a bed net treated with long lasting insecticide and their parents or guardians were instructed in its use. Daily co-trimoxazole prophylaxis was prescribed to all infant participants at enrolment, and to their HIV infected mothers if they were not already taking co-trimoxazole in accordance with national guidelines. HIV infected mothers were advised to exclusively breastfeed their infants until at least 6 months of age and then to wean them to alternative feeding when this was acceptable, safe, feasible, affordable, and sustainable, in accordance with national and WHO guidelines at the time of the study. ${ }^{18}$

\section{Follow up of study participants}

Study participants were followed for all their medical problems in a dedicated study clinic open seven days a week from 8 am to $5 \mathrm{pm}$ with after-hours care available at the Tororo District Hospital adjacent to the study clinic. Parents or guardians were encouraged to avoid using outside medications and to bring their infant to the study clinic or hospital any time the child was ill (transportation costs were reimbursed). Children who presented with new medical problems underwent standardised medical evaluation. A set of algorithms was developed to guide treatment for common illnesses. Drugs with antimalarial activity were avoided for the treatment of non-malarial illnesses when possible.

Monthly routine assessments were done in the study clinic to ensure adherence to the study protocol, drug use, and use of the insecticide treated bed net. Adherence to co-trimoxazole prophylaxis was determined monthly from responses to a validated questionnaire regarding the number of doses taken in the past three days and the percentage of doses taken in the past month. The date of breast feeding cessation was determined from the monthly visits, where children's breastfeeding status and practices were assessed by asking the mothers how they had been feeding their child for the previous seven days. Routine phlebotomy for a complete blood count was performed every 90 days for each participant.

At each follow-up visit, study clinicians assessed participants for adverse events according to standardised criteria based on guidelines from the WHO and National Institutes of Health. Adverse events were defined as untoward medical occurrences, following the guidelines of the International Conference on Harmonization, ${ }^{19}$ and serious adverse events were defined as experiences resulting in death or life threatening events, inpatient hospitalisation, persistent or substantial incapacity, or medical or surgical intervention to prevent serious outcomes. Study participants were withdrawn from the study for permanent movement out of the study area, if they could not be located by study staff for $>60$ consecutive days, withdrawal of informed consent, inability to adhere to the study schedule and procedures, or inability to tolerate study drugs.

Treatment allocation and study drug administration

Six to eight weeks after cessation of breast feeding, each participant was tested for HIV infection by DNA PCR. Participants who acquired HIV during breast feeding were excluded from the study but continued to be followed up and cared for in the clinic as part of another study.

Those who remained HIV uninfected after cessation of breastfeeding were randomised to discontinue cotrimoxazole prophylaxis immediately or to continue daily co-trimoxazole prophylaxis until 2 years of age. A randomisation list was computer generated by an off-site investigator. Sequentially numbered, sealed envelopes containing the prophylaxis assignments were prepared from the randomisation list. The study nurse assigned prophylaxis numbers sequentially and allocated co-trimoxazole prophylaxis or no prophylaxis by opening the envelope corresponding to the prophylaxis number. Participants randomised to continue co-trimoxazole prophylaxis were given a month's supply of the drug at each routine visit (every 30 days).

A nurse administered co-trimoxazole syrup and tablets according to weight based guidelines. Young children weighing $<15 \mathrm{~kg}$ were prescribed 
co-trimoxazole syrup $(40 \mathrm{mg}$ trimethoprim and $200 \mathrm{mg}$ sulfamethoxazole $/ 5 \mathrm{~mL}$ suspension) at the following doses: $2.5 \mathrm{~mL} /$ day for children $\leq 4 \mathrm{~kg}$ in weight, $5 \mathrm{~mL} /$ day for children $>4-8 \mathrm{~kg}$, and $10 \mathrm{~mL} /$ day for children $>8-15 \mathrm{~kg}$. As children reached 10-15 kg, they were switched to co-trimoxazole tablets $(80 \mathrm{mg}$ trimethoprim and $400 \mathrm{mg}$ sulfamethoxazole) and were prescribed one tablet daily thereafter.

\section{Diagnosis and management of malaria}

Medical diagnoses were made by medical officers using predetermined criteria. Participants who presented to the clinic with a documented fever (tympanic temperature $\geq 38.0^{\circ} \mathrm{C}$ ) or history of fever in the previous 24 hours had blood collected by finger prick for a thick blood smear for malaria. If parasites were present, malaria was diagnosed regardless of parasite density. Complicated malaria was diagnosed in participants with a malaria diagnosis and either danger signs or criteria for severe malaria. ${ }^{20}$ Study participants $\geq 4$ months old and weighing $\geq 5 \mathrm{~kg}$ with uncomplicated malaria diagnosed were randomly assigned to receive either open label artemether-lumefantrine or dihydroartemisinin-piperaquine at the time of their first episode of malaria and received the same treatment for all subsequent episodes of uncomplicated malaria diagnosed during the study as part of a nested randomised drug efficacy trial. ${ }^{21}$ Children aged $<4$ months or weighing $<5 \mathrm{~kg}$ with uncomplicated malaria and children with severe or complicated malaria were treated with standard doses of quinine.

Participants with malaria diagnosed were asked to return on days $1,2,3,7,14,21,28$, and any other day they were ill. Follow-up evaluations consisted of a standardised medical history and physical examination. Blood was obtained by finger prick for thick blood smears and storage on filter paper on all follow-up days except day 1 . Treatment outcomes were classified according to WHO guidelines. ${ }^{20}$ Outcomes designated as treatment failures included early treatment failures (complicated malaria or failure to adequately respond to treatment on days 0-3), late clinical failures (complicated malaria or fever and parasitaemia on days 4-28), and late parasitological failures (asymptomatic parasitaemia on days 7-28).

\section{Diagnosis of diarrhoea and respiratory tract infections}

Diarrhoea was included in the analysis if the participant was diagnosed with acute diarrhoea, chronic diarrhoea, dysentery, or gastroenteritis. Acute diarrhoea was defined as more than five watery stools a day for children aged $<1$ year and more than three watery stools a day for children aged $\geq 1$ year of age lasting for less than two weeks. Chronic diarrhoea was defined by the same criteria as acute diarrhoea but lasting for two weeks or longer. Dysentery was defined as grossly bloody or purulent stool by examination or history. Gastroenteritis was defined as diarrhoea and vomiting together of less than four days' duration.
Respiratory tract infections were included in the analysis if the participant was diagnosed with pneumonia or severe pneumonia. Pneumonia was defined as fever and cough with adventitious lung sounds or respiratory distress, or both. Severe pneumonia was defined as pneumonia with severe respiratory distress requiring chest radiography, hospitalisation, and intravenous antibiotics. Upper respiratory tract infections were not included in the analysis.

\section{Laboratory procedures}

Before enrolment, all participants were tested for HIV infection by DNA PCR (Amplicor HIV-1 DNA PCR Test, version 1.5, Roche, Branchburg NJ, USA) using dried blood spots collected on filter paper. Mothers were previously tested at the antenatal clinic by HIV rapid antibody testing or enzyme linked immunosorbent assay (ELISA) antibody testing following national HIV testing algorithms. ${ }^{22}$ All participants were retested at six to eight weeks after complete cessation of breastfeeding with the PCR test, and at 18 months of age with both antibody testing (Determine Rapid test for HIV-1, Abbott Laboratories, Abbott Park IL, USA) and PCR assay of dried blood spots. All positive PCR results were confirmed by repeat bleeding and testing using quantitative HIV RNA PCR (Cobas Amplicor HIV-1 RNA Monitor Test, version 1.5, Roche).

Thick smears were stained with $2 \%$ Giemsa stain for 30 minutes, and parasite density was estimated using a rigorous quality control system previously described ${ }^{6}$ Parasite species was determined on the day that malaria was diagnosed using nested PCR of $18 \mathrm{~S}$ small subunit ribosomal DNA as previously described. ${ }^{23}$ Haemoglobin measurements were made using a portable spectrophotometer (HemoCue, Ängelholm, Sweden) on days 0 and 28 of a given malaria episode or on the day of recurrent malaria.

We randomly selected 148 malaria episodes from each treatment arm divided equally into four periods of six months each (December 2007 to May 2008, June 2008 to November 2008, December 2008 to May 2009, and June 2009 to November 2009) to test for markers of antifolate resistance, as previously described. ${ }^{24-27}$ Using filter paper specimens collected on the day of malaria diagnosis, we tested for the presence of three mutations in the dihydrofolate reductase gene (dhfr $108 \mathrm{~N}, 51 \mathrm{I}$, and 59R) and two mutations in the dihydropteroate synthase gene (dhps $437 \mathrm{G}$ and 540Q) commonly found in East Africa. Additionally, we tested for one dhfr mutation $(164 \mathrm{~L})$ and three dhps mutations (436S, 581G, and 613S) rarely found in Africa, but also associated with antifolate resistance. ${ }^{27}$ Specimens were classified as wild type or pure mutant, or mixed (both mutant and wild type alleles detected in the same specimen).

\section{Statistical analysis}

Data were double entered in Epi-Info, and statistical analysis was performed with Stata version 10 (Stata, 
HIV exposed breastfeeding children enrolled and given co-trimoxazole prophylaxis $(n=203)$

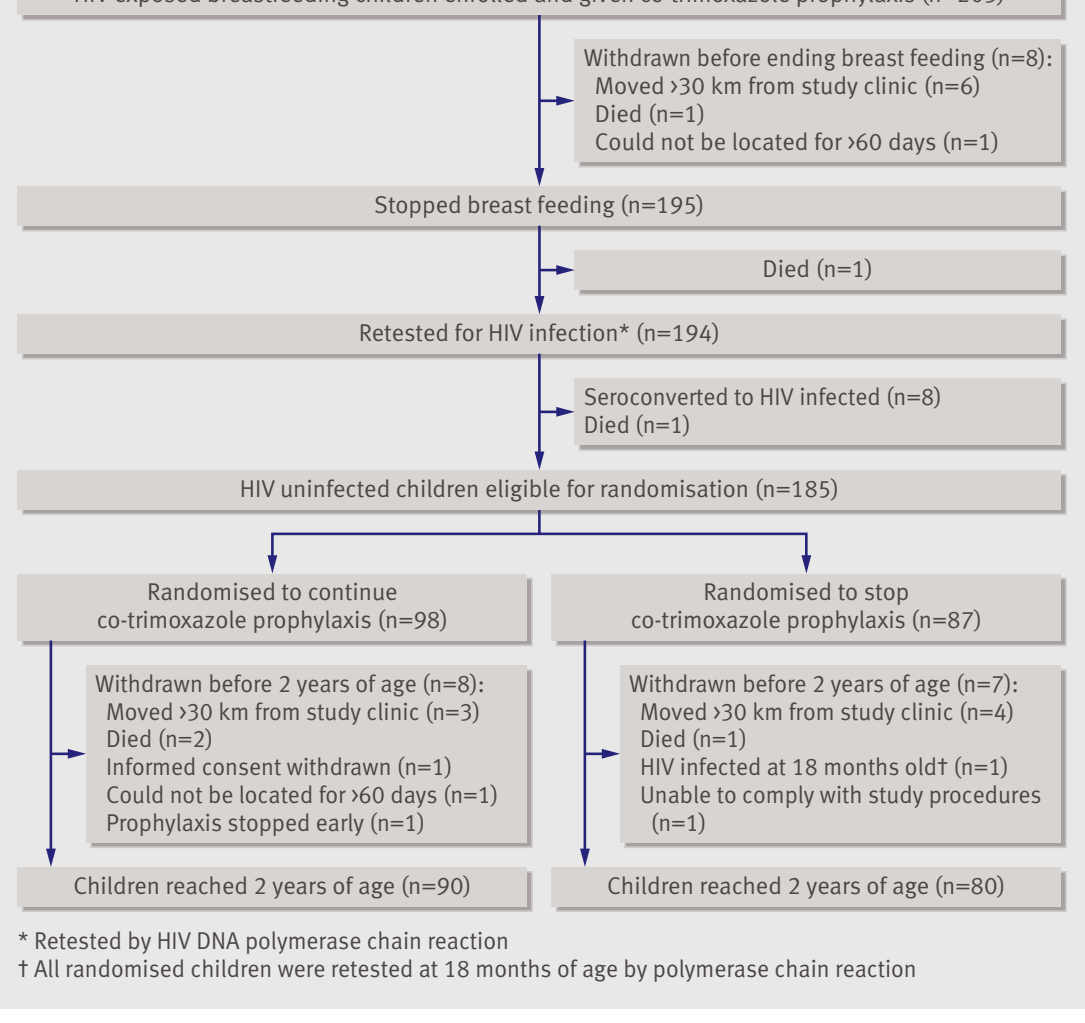

Fig 1| Participant flow through trial

College Station TX, USA). Efficacy and safety data were evaluated based on an intention to treat analysis including all participants who were randomised to continue or discontinue co-trimoxazole prophylaxis. Malaria incidence was defined as the number of new episodes of malaria per person time at risk. Time at risk included time from the day after randomisation until each child reached 2 years of age or was withdrawn from the study, whichever came first. In calculating the incidence of malaria, episodes of malaria and time at risk were censored for 14 days after start of each malaria treatment as children were not at risk for new episodes of malaria during this period. Using molecular genotyping, we have shown that over 95\% of malaria episodes occurring more than 14 days after

Table 1|Baseline characteristics of 185 children at the time of randomisation to continued co-trimoxazole prophylaxis versus no prophylaxis. Values are numbers (percentages) unless stated otherwise

\begin{tabular}{lcc} 
& \multicolumn{2}{c}{ Co-trimoxazole prophylaxis } \\
\cline { 2 - 3 } Characteristic & Continued $(\mathrm{n}=98)$ & Stopped $(\mathrm{n}=\mathbf{8 7})$ \\
Female & $45(46)$ & $47(54)$ \\
\hline Median (interquartile range) age (months): & $3.2(2.3-5.7)$ & $3.9(2.0-6.8)$ \\
\hline At study enrolment & $7.2(6.1-10.0)$ & $7.6(6.8-10.8)$ \\
\hline At stopping breast feeding & $9.6(8.3-12.4)$ & $10.0(8.9-13.5)$ \\
\hline At randomisation & $1.39(1.08$ to 1.76$)$ & $1.32(1.00$ to 1.70$)$ \\
\hline Incidence* $(95 \% \mathrm{Cl})$ of malaria before randomisation & $19(19)$ & $16(18)$ \\
\hline Living in urban area of Tororo town & & \\
\hline${ }^{*}$ No of episodes/person year & & \\
\hline
\end{tabular}

initiation of antimalarial therapy were due to new infections. $^{21}$

Cumulative risks of developing a first episode of malaria after randomisation were estimated with the Kaplan-Meier product limit formula. Data were censored for patients who were prematurely withdrawn from the study. Comparison of survival curves was made using the log rank test. Comparisons of the incidence of malaria, complicated malaria, diarrhoea, respiratory tract infections, hospitalisations, and mortality were made with negative binomial regression, with adjustment for age at randomisation. Protective efficacy was defined using an incidence rate ratio (incidence of malaria among children continuing co-trimoxazole $v$ incidence among those who stopped). We used generalised estimating equation methods with exchangeable correlation structure to account for repeated measures among the same individuals in comparing the association of co-trimoxazole prophylaxis with characteristics of malaria episodes and the presence of the dhfr and dhps mutations. A $\mathrm{P}$ value $<0.05$ was considered significant.

\section{RESULTS}

Trial profile and baseline characteristics

A total of $203 \mathrm{HIV}$ exposed breastfeeding infants were enrolled between August 2007 and April 2008 (fig 1). Ten infants $(5 \%)$ were withdrawn and eight $(4 \%)$ seroconverted to HIV infected status before randomisation. With cessation of breastfeeding, infants of HIV infected mothers were no longer considered to be at risk of acquiring HIV infection. After confirmation of negative HIV status, 185 infants were randomised either to continue co-trimoxazole prophylaxis $(n=98)$ or to stop prophylaxis $(\mathrm{n}=87)$, and a total of $170 \mathrm{com}-$ pleted follow-up (fig 1).

Table 1 shows the participants' baseline characteristics. There were no significant differences between the two study arms in age at randomisation, time at risk and malaria incidence before randomisation, percentage time using the insecticide treated bed net, adherence to continuing or stopping co-trimoxazole after randomisation, or percentage of participants living in Tororo town (associated with a lower incidence of malaria compared with living outside of town).

Protective efficacy of co-trimoxazole prophylaxis against malaria

There were 699 episodes of malaria in total: 299 episodes among participants randomised to continue cotrimoxazole prophylaxis and 400 episodes among those randomised to discontinue co-trimoxazole. Nine episodes were caused by non-falciparum species: five among participants who continued co-trimoxazole prophylaxis (Plasmodium ovale $\mathrm{n}=3$, and $P$ malariae $\mathrm{n}=2$ ) and four among those who stopped co-trimoxazole after breast feeding ( $P$ ovale $\mathrm{n}=2, P$ malariae $\mathrm{n}=1$, and $P$ vivax $\mathrm{n}=1$ ).

Among the 98 infants randomised to continue cotrimoxazole, 72 had at least one episode of malaria 


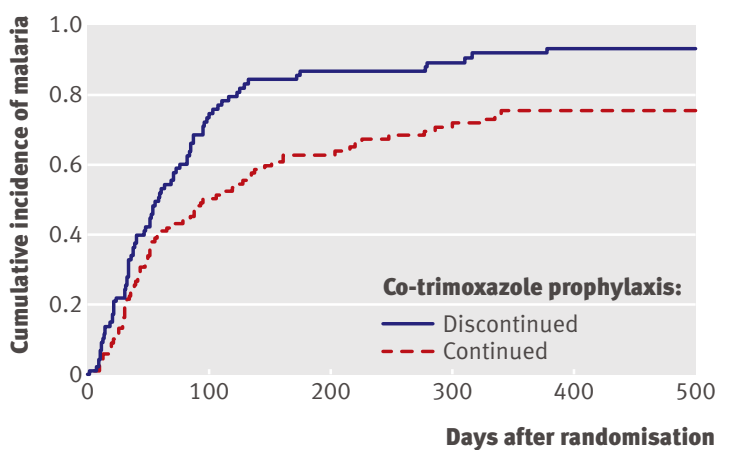

$\overline{\text { Fig } 2}$ | Time to first malaria episode among 185 HIV uninfected children randomised to continued co-trimoxazole prophylaxis versus no prophylaxis $(\mathrm{P}=0.003$ for difference)

during follow-up (cumulative risk 75.7\%). Among the 87 children randomised to stop co-trimoxazole, $78 \mathrm{had}$ at least one episode of malaria during follow-up (cumulative risk 93.4\%). Figure 2 shows that there was a significant difference in the malaria-free survival curves between the two study arms $(\mathrm{P}=0.003)$.

From the time of randomisation until each participant reached 2 years of age, the incidence of malaria was 3.24 episodes/person year (95\% confidence interval 2.89 to 3.63) among participants who continued cotrimoxazole prophylaxis and 5.57 episodes/person year (5.04 to 6.14) among participants who stopped prophylaxis, representing a $39 \%$ lower risk of malaria for the children who continued taking co-trimoxazole
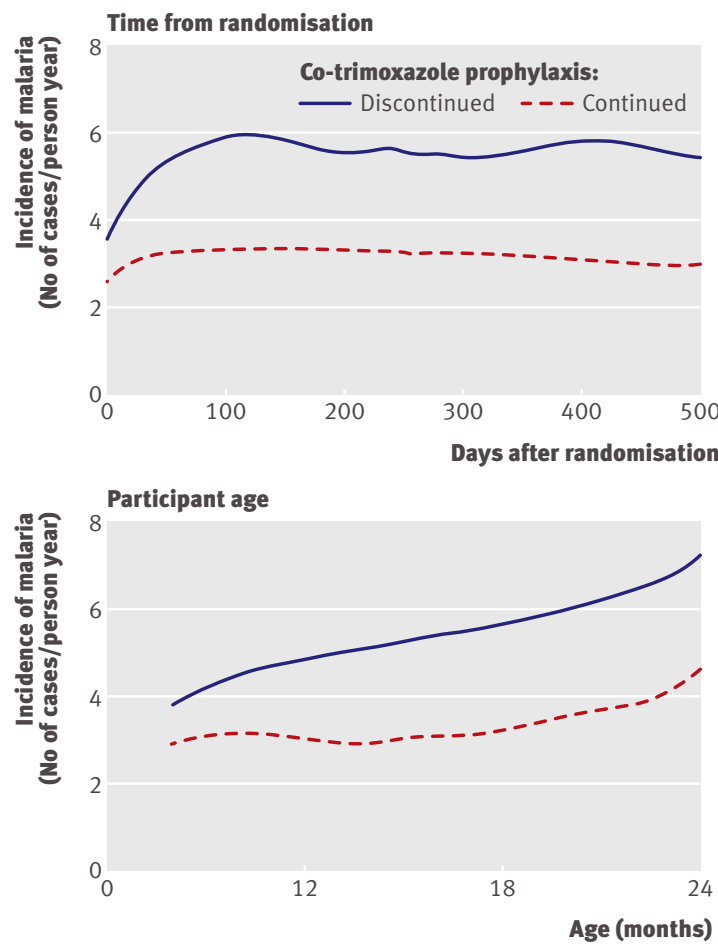

Fig 4 | Protective efficacy of co-trimoxazole prophylaxis against malaria among 185 HIV uninfected children randomised to prophylaxis versus no prophylaxis by time after randomisation and participant age (using locally weighted scatterplot smoothing (LOWESS))

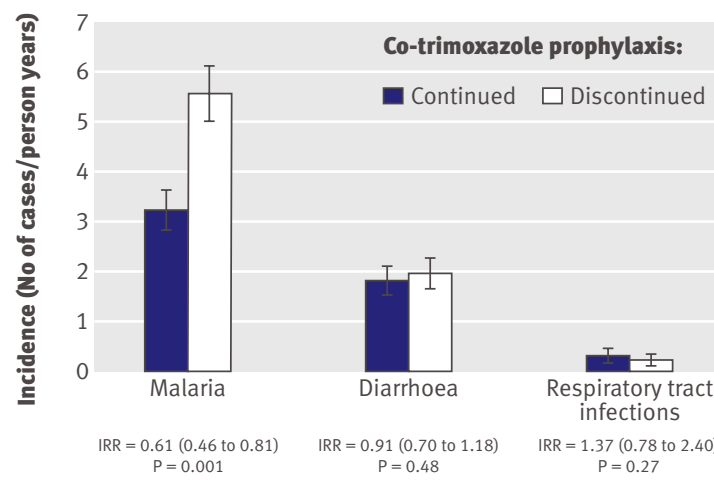

Fig $3 \mid$ Protective efficacy of co-trimoxazole prophylaxis against malaria, diarrhoea, and respiratory tract infections among 185 HIV uninfected children randomised to continued co-trimoxazole prophylaxis versus no prophylaxis. (IRR=incidence rate ratio $(95 \% \mathrm{Cl})$ adjusted for age at randomisation)

(incidence rate ratio 0.61 (95\% confidence interval 0.46 to $0.81, \mathrm{P}=0.001$ ) (fig 3).

In investigating whether the protective effect of cotrimoxazole changed over time, we found there was no difference in the protective efficacy during the first 30 days after randomisation (incidence rate ratio 0.83 (0.45 to 1.53$), \mathrm{P}=0.55)$ compared with the period from 30 days after randomisation to 2 years of age (incidence rate ratio 0.59 (0.44 to 0.80$), \mathrm{P}<0.001)(\mathrm{P}=0.30$ for interaction term). Apart from the period immediately after randomisation, the difference in the incidence of malaria between those children taking co-trimoxazole prophylaxis and those who stopped co-trimoxazole was constant with increasing time after randomisation and with increasing age (fig 4).

There were three episodes of complicated malaria among the participants randomised to continue co-trimoxazole prophylaxis and four episodes among those randomised to stop co-trimoxazole (incidence rate ratio $=0.59, \mathrm{P}=0.50)$. Eight hospitalisations and two deaths occurred among the children randomised to continue co-trimoxazole, compared with four hospitalisations and one death among those randomised to stop co-trimoxazole $(\mathrm{P}=0.79$ and $\mathrm{P}=0.61$, respectively).

Protective efficacy of co-trimoxazole prophylaxis against diarrhoea and respiratory tract infections

From the date of randomisation to 2 years of age, the children randomised to continue co-trimoxazole had 189 episodes of diarrhoea and 33 episodes of respiratory tract infection, compared with 170 episodes of diarrhoea and 20 episodes of respiratory tract infection among those randomised to stop co-trimoxazole.

Children not taking co-trimoxazole had a diarrhoea incidence of 1.96 episodes/person year (95\% confidence interval 1.67 to 2.27 ), whereas those taking cotrimoxazole prophylaxis had a diarrhoea incidence of 1.83 episodes/person year (1.57 to 2.10$)$, resulting in a $9 \%$ protective efficacy that was not significant (incidence rate ratio $0.91, \mathrm{P}=0.48$ ) (fig 3 ). The incidence of respiratory tract infections was 0.32 episodes/person 
Table 2 |Characteristics of the 699 malaria episodes among 185 children randomised to continued co-trimoxazole prophylaxis versus no prophylaxis. Values are numbers (percentages) of episodes unless stated otherwise

\begin{tabular}{|c|c|c|c|}
\hline \multirow[b]{2}{*}{ Characteristic } & \multicolumn{2}{|c|}{$\begin{array}{c}\text { Co-trimoxazole } \\
\text { prophylaxis }\end{array}$} & \multirow[b]{2}{*}{$\begin{array}{l}\text { P value of } \\
\text { difference }\end{array}$} \\
\hline & $\begin{array}{c}\text { Continued } \\
(\mathrm{n}=299)\end{array}$ & $\begin{array}{l}\text { Stopped } \\
(n=400)\end{array}$ & \\
\hline Mean (SD) baseline temperature $\left({ }^{\circ} \mathrm{C}\right)$ & $38.2(1.0)$ & $38.4(1.1)$ & 0.05 \\
\hline Geometric mean baseline parasite density (parasites $/ \mu \mathrm{L}$ ) & 13473 & 21108 & 0.02 \\
\hline Gametocytes present at baseline & $24(8)$ & $22(6)$ & 0.27 \\
\hline Mean (SD) baseline haemoglobin concentration (g/L) & $101(16)$ & $100(15)$ & 0.37 \\
\hline \multicolumn{4}{|l|}{ Malaria treatment regimen: } \\
\hline Artemether-lumefantrine & $163(55)$ & $208(52)$ & - \\
\hline Dihydroartemisinin-piperaquine & $133(44)$ & $184(46)$ & 0.35 \\
\hline Quinine, uncomplicated malaria & 0 & $4(1)$ & - \\
\hline Quinine, complicated malaria & $3(1)$ & $4(1)$ & - \\
\hline \multicolumn{4}{|l|}{ Positive blood smear after treatment: } \\
\hline Day 2 & $26(9)$ & $48(12)$ & 0.14 \\
\hline Day 3 & $1(0.3)$ & $8(2)$ & 0.07 \\
\hline Treatment failure* & 57 (19) & $126(32)$ & 0.006 \\
\hline Mean (SD) haemoglobin recovery† (g/L) & $0.56(1.72)$ & $0.57(1.57)$ & 0.94 \\
\hline
\end{tabular}

*Early treatment failure, late clinical failure, or late parasitological failure according to 28 day WHO outcome classification system.

†Change in haemoglobin from day 0 to day 28 or to day of treatment failure.

year $(0.22$ to 0.45$)$ for participants continuing co-trimoxazole and 0.23 episodes/person year ( 0.14 to 0.36 ) for those not taking co-trimoxazole, resulting in a $37 \%$ increased risk of respiratory tract infections for continued co-trimoxazole, though not statistically significant (incidence rate ratio $1.37, \mathrm{P}=0.27$ ) (fig 3).

\section{Secondary outcomes and adverse events}

At the time of their presentation with malaria, participants randomised to continue taking co-trimoxazole had significantly lower baseline temperature and parasite density than the group who stopped co-trimoxazole (table 2). They also showed a non-significant trend of increased clearance of parasites on days 2 and 3 after malaria diagnosis (table 2). Furthermore, the risk of treatment failure (recurrent parasitaemia by day 28 after diagnosis and treatment) was significantly lower among the children who continued co-trimoxazole $(19 \%$ v 32\%, $\mathrm{P}=0.006)$.

There was no difference between the two study arms in either the median haemoglobin concentration at day 0 of each malaria episode or the mean change in haemoglobin concentration between day 0 and day 28 of malaria follow-up. Median neutrophil values and the prevalence of neutropenia from routine complete blood counts were not significantly different between the two study arms. There were no episodes of skin reactions, allergic drug reactions, or other unexpected adverse events in either study arm.

\section{Markers of antifolate resistance}

Of the 148 malaria episodes randomly selected from each study arm to test for markers of antifolate resistance, we were able to establish genotypes in 139 episodes $(94 \%)$ from the continued co-trimoxazole arm and 142 episodes (96\%) from the discontinued co-trimoxazole arm. Table 3 shows the prevalence of samples with either mixed (both wild type and mutant alleles present) or pure mutant genotypes detected. There was little difference in genotype prevalence between study arms, and most of the mutations detected were in the pure mutant form. There were relatively few mixed mutant infections, with the highest prevalence of mixed infections found at dhfr 59R, where there were $4.3 \%$ mixed infections among episodes in the continued co-trimoxazole arm and 6.3\% mixed infections among episodes in the discontinued co-trimoxazole arm.

The dhfr/dhps quintuple mutant, composed of dhfr 51I, 59R, and $108 \mathrm{~N}$ and dhps $437 \mathrm{G}$ and 540Q mutations, was found in $95 \%$ of episodes from children who continued co-trimoxazole and $91 \%$ of episodes from children who discontinued co-trimoxazole (relative risk 1.05 (0.98 to 1.13 ), $\mathrm{P}=0.17$ ). When examined in four intervals of six months each, from the earliest date of randomisation (December 2007) to the date when the last participant reached 2 years of age (November 2009), the prevalence of the dhfr/dhps quintuple mutant among malaria episodes in the children who continued to take co-trimoxazole was $94 \%$, $89 \%, 100 \%$, and $97 \%$ for each interval, and its prevalence among the children who stopped taking co-trimoxazole was $89 \%, 95 \%, 94 \%$, and $86 \%$. None of the differences between the study arms in the prevalence of the dhfr/dhps quintuple mutant was significant.

\section{DISCUSSION}

\section{Principal findings}

In this study, we found that co-trimoxazole prophylaxis was moderately protective against malaria in HIV exposed children when continued beyond the period of HIV exposure until 2 years of age in an area of high malaria transmission intensity and high prevalence of resistance to antifolate antimalarial drugs. The protective efficacy of co-trimoxazole was lower in the first month after randomisation, but there was not enough power to detect a significant difference between the first month after randomisation and the remainder of the time at risk. Nevertheless, this may indicate a transitional period when children who had stopped taking prophylaxis were still partially protected. Beyond the first month after randomisation, the protective efficacy of co-trimoxazole against malaria was consistent and did not diminish with time or increasing age. Participants who continued co-trimoxazole prophylaxis also had lower parasite densities at the time of malaria diagnosis and a lower prevalence of recurrent parasitaemia by day 28 after diagnosis, suggesting that co-trimoxazole prophylaxis still provided benefit even when children did acquire malaria.

\section{Comparison with other studies}

The protective efficacy of co-trimoxazole prophylaxis against malaria in our trial was substantially lower than 
Table 3|Prevalence of molecular markers of antifolate resistance in 281 episodes of malaria* among 185 children randomised to continued co-trimoxazole prophylaxis versus no prophylaxis. Values are numbers (percentages) of episodes

Co-trimoxazole prophylaxis Continued $(n=139) \quad$ Stopped $(n=142)$

Dihydrofolate reductase gene (dhfr)

\begin{tabular}{lcc}
\hline Ile-51 (51I) & $139(100)$ & $139(98)$ \\
\hline Arg-59 (59R) & $132(95)$ & $131(92)$ \\
\hline Asn-108 (108N) & $139(100)$ & $142(100)$ \\
\hline Leu-164 (164L) & $1(0.7)$ & $1(0.7)$ \\
\hline \multicolumn{2}{l}{ Dihydropteroate synthase gene (dhps) } \\
\hline Ala-436 (436S) & $2(1.4)$ & $2(1.4)$ \\
\hline Gly-437 (437G) & $139(100)$ & $140(99)$ \\
\hline Glu-540 (5400) & $139(100)$ & $141(99)$ \\
\hline Gly-581 (581G) & 0 & 0 \\
\hline Ser-613 (613S) & 0 & 0
\end{tabular}

*Episodes in which Plasmodium falciparum genotypes could be established, from a total of 296 randomly selected episodes.

has been reported in other studies. A randomised controlled trial in Mali showed that co-trimoxazole prophylaxis had a $99.5 \%$ protective efficacy against episodes of clinical malaria and 97\% efficacy against infection. ${ }^{8}$ However, the Mali study enrolled older children (5-15 years old), had a three month followup period, and did not test the HIV status of the children or their mothers, though the overall prevalence of $\mathrm{HIV}$ infection in the Mali study region was $<2 \% \mathrm{com}$ pared with 6\%-7\% in eastern Uganda. ${ }^{28}$ Moreover, sulfadoxine-pyrimethamine, a common antifolate antimalarial drug, was quite efficacious against malaria in most of western Africa at that time, although antifolate resistance is now emerging. ${ }^{2930}$ An observational study in Tororo, Uganda, showed an $80 \%$ decrease in the incidence of malaria among HIV infected adults and children, as well as among the uninfected members of their households, after implementation of cotrimoxazole prophylaxis. ${ }^{79}$ A prospective parallel cohort study in Kampala, Uganda, also showed an $80 \%$ protective efficacy of co-trimoxazole prophylaxis against malaria among HIV infected children compared with HIV uninfected children not taking cotrimoxazole. $^{6}$

The lower protective efficacy of co-trimoxazole observed in our study may be due to an improved study design compared with most of the other studies (randomised trial $v$ observational study), but it was also probably influenced by differences in malaria transmission intensity, participants' HIV status and ages, and prevalence of plasmodial antifolate resistance mutations.

Studies of malaria chemoprophylaxis have been attempted with other drugs, including chloroquine, pyrimethamine and dapsone, chlorproguanil, and atovaquone and proguanil. ${ }^{31-36}$ Despite positive results from some of these studies, routine chemoprophylaxis for malaria has never been widely implemented because of logistical and economic constraints, and the concern for rebound of malaria incidence after cessation of chemoprophylaxis. Co-trimoxazole prophylaxis may have a logistical advantage over other antimalarial prophylactic drugs since it is already in widespread use among HIV infected and HIV exposed patients in many countries, though the issue of rebound malaria after cessation needs to be investigated.

The protective efficacy of daily co-trimoxazole prophylaxis against malaria in our study was comparable to that reported in trials of the RTS,S/AS01 malaria vaccine and intermittent preventive therapy in infants (IPTi). ${ }^{37-42}$ The IPTi Consortium has completed six clinical trials investigating the use of such therapy with sulfadoxine-pyrimethamine in different settings and showed an average protective efficacy of $30 \%$ with three trials, as well as reducing anaemia. ${ }^{40}$ The IPTi trials differ from our trial in that a lower percentage of participants used bed nets, the risk of malaria was substantially lower, and the efficacy of sulfadoxine-pyrimethamine treatment for uncomplicated malaria was greater than $70 \%$. It is likely that the protective efficacy of intermittent preventive therapy with sulfadoxine-pyrimethamine will decrease with increasing antifolate resistance, and a replacement drug has not yet been firmly established. ${ }^{43}$ In our trial co-trimoxazole prophylaxis showed a protective efficacy against malaria of 39\%, even in the setting of high antifolate resistance and high malaria risk in Tororo. However, this level of protection from malaria comes at the expense of the increased potential for adverse events, cost, and logistical difficulties of providing daily co-trimoxazole compared with intermittent sulfadoxinepyrimethamine prescribed at the time of childhood vaccinations.

\section{Antifolate resistance and secondary outcomes}

Because of in vitro evidence of cross resistance to cotrimoxazole and sulfadoxine-pyrimethamine, ${ }^{445}$ scientists have been concerned that the widespread use of co-trimoxazole prophylaxis will result in an increased prevalence of sulfadoxine-pyrimethamine resistant parasites. ${ }^{146}$ Recent studies in Tororo and Kampala, Uganda, of HIV infected children and adults taking daily co-trimoxazole prophylaxis have found no association between co-trimoxazole use and increased prevalence of mutations conferring antifolate resistance. ${ }^{4748}$ Both studies were limited by a high baseline level of antifolate resistant genotypes and relatively small numbers. In our study, the prevalence of the dhfr/dhps quintuple mutant was similar in the two treatment arms, but our ability to detect an association between co-trimoxazole use and the dhfr/ dhps quintuple mutant was limited by the virtual saturation of the quintuple mutant in the parasite population around Tororo. Indeed, to our knowledge, the overall prevalence of the dhfr/dhps quintuple mutant in our study is the highest yet reported in Africa. Although the dhfr/dhps quintuple mutant has been shown to be related to the failure of sulfadoxine-pyrimethamine therapy for uncomplicated malaria, ${ }^{2549} 50$ 


\section{WHAT IS ALREADY KNOWN ON THIS TOPIC}

Malaria kills about 1 million people each year, $90 \%$ of whom are children under 5 years old living in sub-Saharan Africa

Observational studies of daily co-trimoxazole prophylaxis in children and adults have shown a protective efficacy of $80 \%$ and higher against malaria

\section{WHAT THIS STUDY ADDS}

This is the first randomised controlled trial of co-trimoxazole prophylaxis against malaria in HIV exposed children, set in rural Uganda, where both malaria transmission intensity and resistance to antimalarial antifolate drugs are high

Continuing daily co-trimoxazole prophylaxis for HIV exposed children after cessation of breast feeding to 2 years of age decreased the incidence of malaria by $39 \%$ compared with stopping prophylaxis at end of breast feeding

the role of this mutant in affecting the protective efficacy of co-trimoxazole prophylaxis against malaria has yet to be determined.

HIV exposed children experience increased morbidity and mortality in their first years of life compared with HIV uninfected children born to uninfected mothers. ${ }^{355152}$ The reasons for these poor outcomes among HIV exposed children have been clearly postulated $^{5354}$ and may explain why co-trimoxazole prophylaxis in this population could provide protection against serious bacterial and parasitic infections. However, although co-trimoxazole prophylaxis was protective against malaria in our cohort, we detected no differences between the two study arms in the rates of either diarrhoea or respiratory tract infections, two common and potentially fatal infectious syndromes associated with HIV infection. The question of whether the benefits of co-trimoxazole prophylaxis among HIV exposed infants who remain HIV uninfected outweigh the risks has yet to be clearly delineated.

\section{Conclusions and policy implications}

Our data add to the growing number of malaria chemoprevention trials, which are trying to discern the best drug, dose, treatment frequency, and treatment duration for children living in areas of differing malaria transmission intensities. The primary relevance of our findings is that co-trimoxazole prophylaxis afforded significant protection against malaria in a setting of highly endemic and highly resistant malaria. Currently, co-trimoxazole is used as prophylaxis in HIV exposed children to prevent opportunistic infections such as toxoplasmosis and pneumocystis. Given the increasing interest in chemoprevention of malaria, we looked at continuing co-trimoxazole prophylaxis among those children who did not seroconvert during breast feeding, and we found that continuing co-trimoxazole helps to prevent malaria in this population. Our study also provides insight into providing malaria chemoprevention to all children, regardless of HIV status or exposure.

It is premature to conclude whether co-trimoxazole should be recommended for HIV exposed children testing HIV negative after cessation of breast feeding.
Differences in malaria transmission intensity and prevalence of antifolate resistance could affect the protective efficacy of co-trimoxazole prophylaxis at different sites. Furthermore, questions remain over the effect of co-trimoxazole use on the continued development of antifolate resistance and prevention of acquisition of natural antimalarial immunity, which may result in an increased rebound incidence of malaria upon cessation of prophylaxis. To help answer some of these questions, we are following our cohort to 5 years of age to examine the continued protective efficacy of co-trimoxazole against malaria and to evaluate rebound effects after withdrawal of co-trimoxazole. The results of this and other studies ${ }^{55}$ should better inform the use of co-trimoxazole prophylaxis in HIV exposed infants in areas with endemic malaria.

We thank the clinical study team and our administrative staff. We are grateful to the children who participated in this study and to their parents and guardians.

Contributors: TGS, JH, GD, and JWT developed the concept of the study. All authors were involved with writing the protocol. TS, JH, GD, and JWT were responsible for data analysis. TS had full access to all the data in the study, takes responsibility for the integrity of the data and accuracy of the data analysis, and is the guarantor.

Funding: Participants in this study were enrolled in programmes supported by the US President's Emergency Plan for AIDS Relief and by Cooperative Agreement No U62P024421 from the Centers for Disease Control and Prevention (CDC); National Center for HIV, Viral Hepatitis, STD, and TB Prevention (NCHHSTP); and Global AIDS Program (GAP). Funding was also provided by the Doris Duke Charitable Foundation (GD is a recipient of the Clinical Scientist Development Award, and NV is a recipient of the Clinical Research Fellowship). TS was funded through the Puget Sound Partners in Global Health and NIH/NIAID K23-AI082553. The funders were not involved with study design, data analysis, or manuscript preparation. The contents of the manuscript are solely the responsibility of the authors and do not necessarily represent the official views of the CDC or Doris Duke Charitable Foundation.

Competing interests: All authors have completed the Unified Competing Interest form at www.icmje.org/coi_disclosure.pdf (available on request from the corresponding author) and declare: no support from any organisation for the submitted work, no financial relationships with any organisations that might have an interest in the submitted work in the previous three years, and no other relationships or activities that could appear to have influenced the submitted work.

Ethical approval: The study protocol was approved by the Uganda National Council of Science and Technology and the institutional review boards of the University of California San Francisco, Makerere University, the University of Washington, and the Centers for Disease Control and Prevention.

Data sharing: No additional data available.

1 World Health Organization \& Roll Back Malaria. Malaria and HIV interactions and their implications for public health policy. Report of a technical consultation, Geneva, Switzerland, 23-5 June 2004. 2010. www.who.int/hiv/pub/prev_care/malariahiv.pdf.

2 Joint United Nations Programme on HIV/AIDS, (UNAIDS) \& World Health Organization (WHO). AIDS epidemic update: November 2009. UNAIDS and WHO, 2009.

3 Brahmbhatt H, Kigozi G, Wabwire-Mangen F, Serwadda D, Lutalo T, Nalugoda F, et al. Mortality in HIV-infected and uninfected children of HIV-infected and uninfected mothers in rural Uganda. J Acquir Immune Defic Syndr 2006;41:504-8.

4 Crampin AC, Floyd S, Glynn JR, Madise N, Nyondo A, Khondowe MM, et al. The long-term impact of HIV and orphanhood on the mortality and physical well-being of children in rural Malawi. Aids 2003;17:389-97.

5 Kuhn L, Kasonde P, Sinkala M, Kankasa C, Semrau K, Scott N, et al. Does severity of HIV disease in HIV-infected mothers affect mortality and morbidity among their uninfected infants? Clin Infect Dis 2005;41:1654-61.

6 Kamya MR, Gasasira AF, Achan J, Mebrahtu T, Ruel T, Kekitiinwa A, et al. Effects of trimethoprim-sulfamethoxazole and insecticide- 
treated bednets on malaria among HIV-infected Ugandan children. Aids 2007;21:2059-66

7 Mermin J, Ekwaru JP, Liechty CA, Were W, Downing R, Ransom R, et al. Effect of co-trimoxazole prophylaxis, antiretroviral therapy, and insecticide-treated bednets on the frequency of malaria in HIV-1infected adults in Uganda: a prospective cohort study. Lancet 2006;367:1256-61.

8 Thera MA, Sehdev PS, Coulibaly D, Traore K, Garba MN, Cissoko Y, et al. Impact of trimethoprim-sulfamethoxazole prophylaxis on falciparum malaria infection and disease. I Infect Dis 2005;192:1823-9.

9 Mermin J, Lule J, Ekwaru JP, Downing R, Hughes P, Bunnell R, et al. Cotrimoxazole prophylaxis by HIV-infected persons in Ugand reduces morbidity and mortality among HIV-uninfected family members. Aids 2005;19:1035-42.

10 World Health Organization. Guidelines on cotrimoxazole prophylaxis for HIV-related infections among children, adolescents and adults: recommendations for a public health approach. WHO, 2006.

11 Anglaret X, Chene G, Attia A, Toure S, Lafont S, Combe P, et al. Early chemoprophylaxis with trimethoprim-sulphamethoxazole for HIV-1 infected adults in Abidjan, Cote d'Ivoire: a randomised trial. CotrimoCl Study Group. Lancet 1999;353:1463-8.

12 Chintu C, Bhat GJ, Walker AS, Mulenga V, Sinyinza F, Lishimpi K, et a Co-trimoxazole as prophylaxis against opportunistic infections in HIV-infected Zambian children (CHAP): a double-blind randomised placebo-controlled trial. Lancet 2004;364:1865-71.

13 Wiktor SZ, Sassan-Morokro M, Grant AD, Abouya L, Karon JM, Maurice C, et al. Efficacy of trimethoprim-sulphamethoxazole prophylaxis to decrease morbidity and mortality in HIV-1-infected patients with tuberculosis in Abidjan, Cote d'lvoire: a randomised controlled trial. Lancet 1999;353:1469-75.

14 Gill C), Sabin LL, Tham J, Hamer DH. Reconsidering empirical cotrimoxazole prophylaxis for infants exposed to HIV infection. Bull World Health Organ 2004;82:290-7.

15 Santmyire BR. Vertical transmission of HIV from mother to child in sub-Saharan Africa: modes of transmission and methods for prevention. Obstet Gynecol Surv 2001;56:306-12.

16 Wilfert CM, Fowler MG. Balancing maternal and infant benefits and the consequences of breast-feeding in the developing world during the era of HIV infection. / Infect Dis 2007;195:165-7.

17 Okello PE, Van Bortel W, Byaruhanga AM, Correwyn A, Roelants P, Talisuna $A$, et al. Variation in malaria transmission intensity in seven sites throughout Uganda. Am / Trop Med Hyg 2006;75:219-25.

18 World Health Organization. WHO guidelines on infant feeding: principles and recommendations for infant feeding in the context of HIV and a summary of the evidence. 2010. http://whqlibdoc.who. int/publications/2010/9789241599535_eng.pdf.

19 International Conference on Harmonisation. ICH harmonised tripartite guideline. Clinical safety data management: definitions and standards for expedited reporting E2A. ICH, 1994.

20 World Health Organization. WHO guidelines for the treatment of malaria, 2006. WHO, 2006.

21 Arinaitwe E, Sandison TG, Wanzira H, Kakuru A, Homsy J, Kalamya J, et al. Artemether-lumefantrine versus dihydroartemisinin piperaquine for falciparum malaria: a longitudinal, randomized tria in young Ugandan children. Clin Infect Dis 2009;49:1629-37.

22 Uganda Ministry of Health. Uganda national policy guidelines for HIV voluntary counselling and testing. Uganda Ministry of Health, 2005

23 Snounou G, Viriyakosol S, Jarra W, Thaithong S, Brown KN. Identification of the four human malaria parasite species in field samples by the polymerase chain reaction and detection of a high prevalence of mixed infections. Mol Biochem Parasitol 1993;58:283-92.

24 Duraisingh MT, Curtis J, Warhurst DC. Plasmodium falciparum: detection of polymorphisms in the dihydrofolate reductase and dihydropteroate synthetase genes by PCR and restriction digestion. Exp Parasitol 1998;89:1-8.

25 Kyabayinze D, Cattamanchi A, Kamya MR, Rosenthal PJ, Dorsey G. Validation of a simplified method for using molecular markers to predict sulfadoxine-pyrimethamine treatment failure in African children with falciparum malaria. Am J Trop Med Hyg 2003;69:247-52.

26 Plowe CV, Diimde A, Bouare M, Doumbo O, Wellems TE. Pyrimethamine and proguanil resistance-conferring mutations in Plasmodium falciparum dihydrofolate reductase: polymerase chain reaction methods for surveillance in Africa. Am J Trop Med Hyg 1995;52:565-8

27 Sibley CH, Hyde JE, Sims PF, Plowe CV, Kublin JG, Mberu EK, et al. Pyrimethamine-sulfadoxine resistance in Plasmodium falciparum: what next? Trends Parasitol 2001;17:582-8.

28 AIDS epidemic update: December 2005, Sub-Saharan Africa. 2005 www.unaids.org/epi/2005/doc/EPlupdate2005_pdf_en/ Epi05_05_en.pdf.

29 Nahum A, Erhart A, Ahounou D, Bonou D, Van Overmeir C, Menten J, et al. Extended high efficacy of the combination sulphadoxine- pyrimethamine with artesunate in children with uncomplicated falciparum malaria on the Benin coast, West Africa. Malar J 2009;8:37.

30 Tinto H, Ouedraogo JB, Zongo I, van Overmeir C, van Marck E, Guiguemde TR, et al. Sulfadoxine-pyrimethamine efficacy and selection of Plasmodium falciparum DHFR mutations in Burkina Faso before its introduction as intermittent preventive treatment for pregnant women. Am J Trop Med Hyg 2007;76:608-13.

31 Faucher JF, Binder R, Missinou MA, Matsiegui PB, Gruss H, Neubauer R, et al. Efficacy of atovaquone/proguanil for malaria prophylaxis in children and its effect on the immunogenicity of live oral typhoid and cholera vaccines. Clin Infect Dis 2002;35:1147-54.

32 Greenwood BM, Greenwood AM, Bradley AK, Snow RW, Byass P, Hayes RJ, et al. Comparison of two strategies for control of malaria within a primary health care programme in the Gambia. Lancet 1988;1:1121-7.

33 Hogh B, Thompson R, Lobo V, Dgedge M, Dziegiel M, Borre M, et al. The influence of Maloprim chemoprophylaxis on cellular and humoral immune responses to Plasmodium falciparum asexual blood stage antigens in schoolchildren living in a malaria endemic area of Mozambique. Acta Trop 1994;57:265-77.

34 Menendez C, Kahigwa E, Hirt R, Vounatsou P, Aponte JJ, Font F, et al. Randomised placebo-controlled trial of iron supplementation and malaria chemoprophylaxis for prevention of severe anaemia and malaria in Tanzanian infants. Lancet 1997;350:844-50.

35 Watkins WM, Brandling-Bennett AD, Oloo AJ, Howells RE, Gilles HM, Koech DK. Inadequacy of chlorproguanil $20 \mathrm{mg}$ per week as chemoprophylaxis for falciparum malaria in Kenya. Lancet 1987;1:125-8.

36 Wolde B, Pickering J, Wotton K. Chloroquine chemoprophylaxis in children during peak transmission period in Ethiopia. J Trop Med Hyg 1994;97:215-8.

37 Alonso PL, Sacarlal J, Aponte JJ, Leach A, Macete E, Aide P, et al. Duration of protection with RTS,S/AS02A malaria vaccine in prevention of Plasmodium falciparum disease in Mozambican children: single-blind extended follow-up of a randomised controlled trial. Lancet 2005;366:2012-8.

38 Alonso PL, Sacarlal J, Aponte J, Leach A, Macete E, Milman J, et al. Efficacy of the RTS,S/AS02A vaccine against Plasmodium falciparum infection and disease in young African children: randomised controlled trial. Lancet 2004;364:1411-20.

39 Aponte JJ, Aide P, Renom M, Mandomando I, Bassat Q, Sacarlal J, et al. Safety of the RTS,S/AS02D candidate malaria vaccine in infants living in a highly endemic area of Mozambique: a double blind randomised controlled phase I/Ilb trial. Lancet 2007;370:1543-51.

40 Aponte JJ, Schellenberg D, Egan A, Breckenridge A, Carneiro I, Critchley J, et al. Efficacy and safety of intermittent preventive treatment with sulfadoxine-pyrimethamine for malaria in African infants: a pooled analysis of six randomised, placebo-controlled trials. Lancet 2009;374:1533-42.

41 Bejon P, Lusingu J, Olotu A, Leach A, Lievens M, Vekemans J, et al. Efficacy of RTS,S/AS01E vaccine against malaria in children 5 to 17 months of age. N Engl J Med 2008;359:2521-32.

42 Sacarlal J, Aide P, Aponte J,, Renom M, Leach A, Mandomando I, et al. Long-term safety and efficacy of the RTS,S/AS02A malaria vaccine in Mozambican children. J Infect Dis 2009;200:329-36.

43 Cairns M, Gosling R, Carneiro I, Gesase S, Mosha JF, Hashim R, et al. Duration of protection against clinical malaria provided by three regimens of intermittent preventive treatment in Tanzanian infants. PloS One 2010;5:e9467.

44 Iyer JK, Milhous WK, Cortese JF, Kublin JG, Plowe CV. Plasmodium falciparum cross-resistance between trimethoprim and pyrimethamine. Lancet 2001;358:1066-7.

45 Triglia T, Menting JG, Wilson C, Cowman AF. Mutations in dihydropteroate synthase are responsible for sulfone and sulfonamide resistance in Plasmodium falciparum. Proc Natl Acad Sci USA 1997;94:13944-9.

46 Lynen L, Jacobs J, Colebunders R. Co-trimoxazole prophylaxis in tropical countries in the era of highly active antiretroviral therapy: do we know enough? Trans R Soc Trop Med Hyg 2007;101:1059-60.

47 Gasasira AF, Kamya MR, Ochong EO, Vora N, Achan J, Charlebois E, et al. Effect of trimethoprim-sulphamethoxazole on the risk of malaria in HIV-infected Ugandan children living in an area of widespread antifolate resistance. Malar J 2010;9:177

48 Malamba S, Sandison T, Lule J, Reingold A, Walker J, Dorsey G, et al. Plasmodium falciparum dihydrofolate reductase and dihyropteroate synthase mutations and the use of trimethoprim-sulfamethoxazole prophylaxis among persons infected with human immunodeficiency virus. Am J Trop Med Hyg 2010;82:766-71.

49 Dorsey G, Dokomajilar C, Kiggundu M, Staedke SG, Kamya MR, Rosenthal PJ. Principal role of dihydropteroate synthase mutations in mediating resistance to sulfadoxine-pyrimethamine in single-drug and combination therapy of uncomplicated malaria in Uganda. Am J Trop Med Hyg 2004;71:758-63. 
50 Kublin JG, Dzinjalamala FK, Kamwendo DD, Malkin EM, Cortese JF, Martino LM, et al. Molecular markers for failure of sulfadoxinepyrimethamine and chlorproguanil-dapsone treatment of Plasmodium falciparum malaria. J Infect Dis 2002;185:380-8.

51 Chatterjee A, Bosch RJ, Hunter DJ, Fataki MR, Msamanga GI, Fawzi WW. Maternal disease stage and child undernutrition in relation to mortality among children born to HIV-infected women in Tanzania. J Acquir Immune Defic Syndr 2007;46:599-606.

52 Newell ML, Coovadia H, Cortina-Borja M, Rollins N, Gaillard P, Dabis F. Mortality of infected and uninfected infants born to HIVinfected mothers in Africa: a pooled analysis. Lancet 2004;364:1236-43.
53 Kuhn L, Thea DM, Aldrovandi GM. Bystander effects: children who escape infection but not harm. J Acquir Immune Defic Syndr 2007;46:517-8.

54 Marinda E, Humphrey JH, Iliff PJ, Mutasa K, Nathoo KJ, Piwoz EG, et al. Child mortality according to maternal and infant HIV status in Zimbabwe. Pediatr Infect Dis / 2007;26:519-26.

55 McIntyre J. Use of antiretrovirals during pregnancy and breastfeeding in low-income and middle-income countries. Curr Opin HIV AIDS;5:48-53.

Accepted: 04 January 2011 